\title{
High-Resolution NMR Spectra and Conformation of Poly- (vinylidene fluoride) and Their Relation with Crystal Modifications
}

\author{
Yoshiharu ToIDA and Riichirô CHûsô \\ Department of Polymer Chemistry, Tokyo Institute of \\ Technology, Meguro-ku, Tokyo 152, Japan.
}

(Received July 23, 1973)

\begin{abstract}
KEY WORDS NMR / Poly(vinylidene fluoride) / Conformation / Crystallization /
\end{abstract}

It is well known that the crystal structure of polymers depends on the species of the solvent in crystallization. However, few investigations have been reported concerning the relation of the polymer conformation in solvent with its crystal forms. The reason why such investigations have not been carried out is that there are very few methods to study the microstructure of polymers in solution. NMR, however, may serve to determine such structure.

Poly(vinylidene fluoride) (PVDF) has two or three crystal modifications, ${ }^{1-3}$ i.e., the $T G T \bar{G}$ conformation ( $\alpha$-type) and the TT conformation ( $\beta$-type), which can be obtained selectively by a variety of factors including the species of solvent, the concentrations of polymers in crystallization, and the annealing temperature.

The crystals obtained by recrystallization in dimethylsulfoxide (DMSO) are exclusively $\beta$-type, while in the mixed solvent system of monochlorobenzene (MCB)-DMSO, the crystal forms are richer in $\alpha$-type with increase of the fraction of $\mathrm{MCB}^{2}$. We investigated the molecular conformation of PVDF in these mixed solvent systems with the aid of NMR spectra.

The proton NMR spectra of PVDF consist of two parts, corresponding to methylene protons with head-to-tail and head-to-head bondings. ${ }^{4}$ (In this paper $-\mathrm{CF}_{2}-$ and $-\mathrm{CH}_{2}-$ will be regarded as the head and tail of the monomer units, respectively.) The former consists of a quintet, coupled with four vicinal fluorines of the protons in question. The coupling constants of the $\mathrm{H}-\mathrm{F}$ depend on the conformation of molecules, that is, the coupling constants observed are an average value at three energically favored forms around the skeletal $\mathrm{C}-\mathrm{C}$ bond. Then the observed coupling constant is given by

$$
(J)_{\text {obs }}=\frac{\left(J_{T}+3 J_{G}\right)+\left(J_{T}+J_{G}\right) \exp (-E / R T)}{2[2+\exp (-E / R T)]}
$$

where $E$ is the energy difference between the trans and gauche conformations with respect to the skeletal $\mathrm{C}-\mathrm{C}-\mathrm{C}-\mathrm{C}$ bonds. $J_{T}$ and $J_{G}$ are vicinal coupling constants between $\mathrm{H}$ and $\mathrm{F}$, and the subscripts $T$ and $G$ denote trans and gauche forms with respect to the $\mathrm{H}-\mathrm{C}-\mathrm{C}-\mathrm{F}$ bonds.

From the temperature dependence of $(J)_{\mathrm{obs}}$, $J_{T}, J_{G}$, and $E$ are obtained as $54.6 \mathrm{~Hz},-0.6 \mathrm{~Hz}$, and $410 \mathrm{cal} / \mathrm{mol}$ for the $10-\% \mathrm{wt}$ solution of PVDF in DMSO (Figure 1), for temperatures between 60 and $140^{\circ} \mathrm{C}$. The values of $J_{G}$ and $J_{T}$

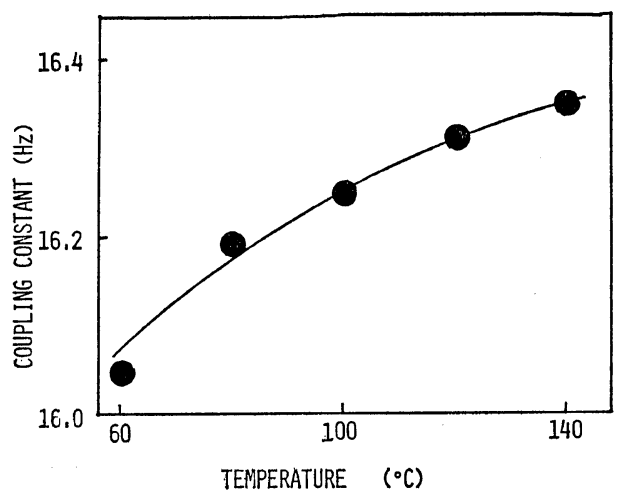

Figure 1. The temperature dependence of $(J)_{\text {obs }}$ of PVDF in DMSO (10-wt \% solution). 


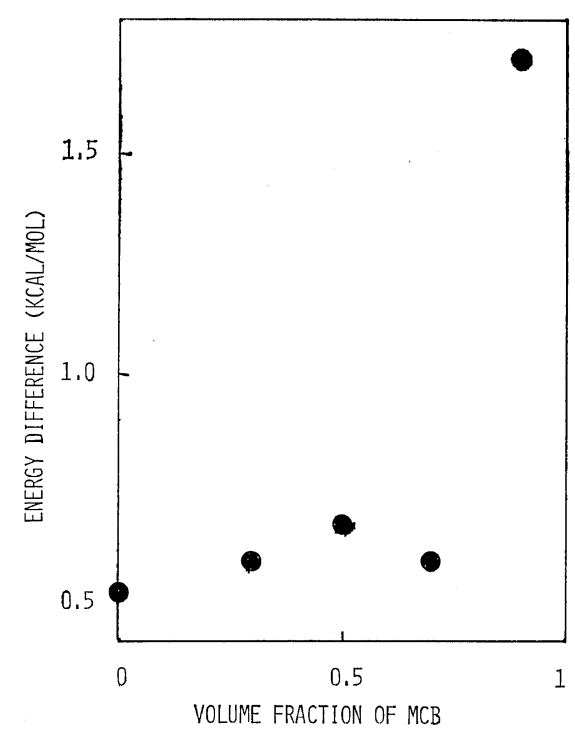

Figure 2. Variation of energy difference $E$ with solvent (1-wt \% solution).

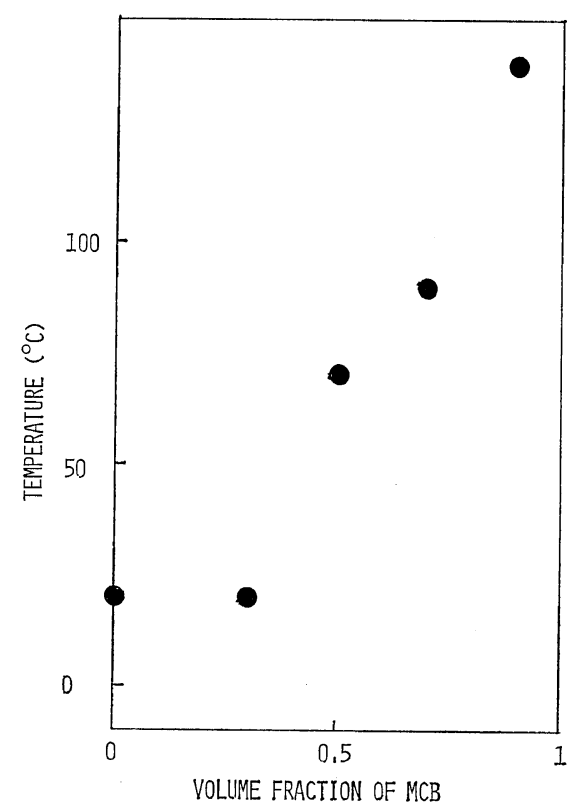

Figure 3. Variation of crystallization temperature with solvent (1-wt \% solution).

do not agree well with these for low molecular weight compounds with similar structures. However, the argument given below does not depend strongly on the values of $J_{G}$ and $J_{T}$.

With the above-obtained values of $J_{T}$ and $J_{G}$,

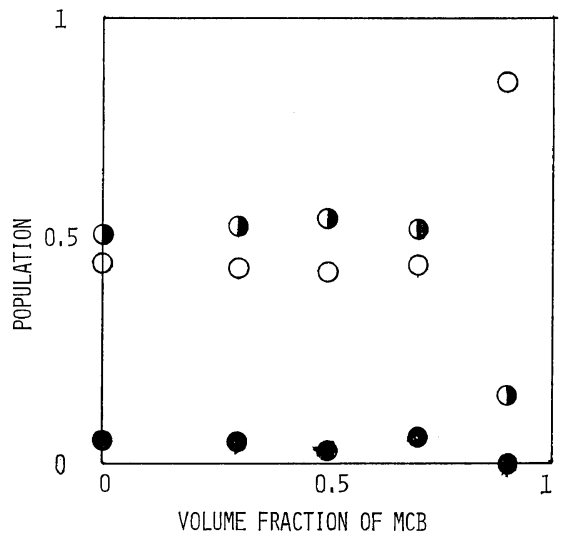

Figure 4. Variation of $\boldsymbol{P}_{T T}(\bullet), \boldsymbol{P}_{T G}(\bigcirc)$, and $\boldsymbol{P}_{G G}$ (O) with solvent (1-wt \% solution).

one can get values of $E$ for the 1-wt $\%$ solution of PVDF in MCB-DMSO mixed solvent systems. These values are also independent of temperature, but they are strongly affected by the volume fraction of $\mathrm{MCB}$ in the mixed solvent. The values of $E$ are shown in Figure 2.

Crystals precipitate at different temperatures in these systems for different fractions of MCB. Crystallization temperature are shown in Figure 3.

Population of $T T, T G$, and $G G$ conformers at crystallization temperatures are calculated under the assumption of Bernouilli trials and these are plotted against the volume fraction of MCB (Figure 4).

In the systems of higher MCB fraction the energy difference between two conformations becomes extremely large. This is one of the reasons why one cannot get any $\beta$-type crystals from the solvents with higher MCB fraction. So Figure 4 is one possible explanation for the difference of crystal structures of PVDF with the fraction of $\mathrm{MCB}$ in the above mixed solvent systems. That is to say, the crystals with $T T$ conformation precipitate if a small amount of $T T$ conformation can exist, while the crystal with $T G$ conformation (which is a part of $T G T \bar{G}$ one) precipitates if the $T T$ conformation can exist only in an extremely small fraction. Other factors must be taken into account for a quantitative explanation.

Crystal forms of polymers are determined not only by the short range interaction in a segment but also by intramolecular and/or intermolecular 
interaction between segments spacially close to each other but with large contour lengths. The extended conformation is favorable if such an interaction is taken into account. Of the three conformations $T T, T G$, and $G G$, the $T T$ is the most extended one. In the system of lower fraction of MCB, there is a small but finite probability for the occurence of $T T$ conformation, thus $T T$ can become a nucleus of crystallization and $\beta$-type crystals are produced, whereas in the systems of higher fraction of MCB, because of the small probability of $T T$ conformation, the next favorable one, the $T G$ conformation becomes the nucleus of the crystallization and $\alpha$ type crystals are produced. Measurement of the higher-order parameter will make such discussions more certain.
The authors wish to express their sincere thanks to Dr. K. Okuda (Tokyo Laboratory, Kureha Chemical Industry Co., Ltd.) for his kind courtesy in supplying the samples, and Mr. M. Kambe (our laboratory) for his help in measuring the NMR spectroscopy.

\section{REFERENCES}

1. S. S. Leshchenko, V. L. Karpov, and V. A. Karrgin, Vysokomol. Soedin., 1, 1538 (1959).

2. K. Okuda, T. Yoshida, M. Sugita, and M. Asahina, J. Polym. Sci., Part B, 5, 465 (1967).

3. R. Hasegawa, Y. Takahashi, Y. Chatani, and H. Tadokoro, Polymer J., 3, 600 (1972).

4. C. W. Wilson, III, and E. R. Santee, Jr., J. Polym. Sci., Part C, 8, 97 (1965). 